\title{
Tricking the tumour microenvironment into becoming our best rational drug design factory: reversal of immune suppression
}

\author{
Martin L. Ashdown \\ Faculty of Medicine, Dentistry and Health Sciences, University of Melbourne, Melbourne 3011, Australia. \\ Correspondence to: Martin L. Ashdown, Faculty of Medicine, Dentistry and Health Sciences, University of Melbourne, \\ Melbourne 3011, Australia. E-mail: ashdownm@unimelb.edu.au \\ How to cite this article: Ashdown ML. Tricking the tumour microenvironment into becoming our best rational drug design \\ factory: reversal of immune suppression. J Cancer Metastasis Treat 2022;8:6. https://dx.doi.org/10.20517/2394-4722.2022.01
}

Received: 4 Jan 2022 First Decision: 10 Feb 2022 Revised: 17 Feb 2022 Accepted: 21 Feb 2022 Published: 28 Feb 2022

Academic Editors: Lucio Miele, Steven Fiering Copy Editor: Xi-Jun Chen Production Editor: Xi-Jun Chen

\begin{abstract}
The immune cellular components of the tumour microenvironment are a diverse group of cells that paradoxically are now appreciated to have a coordinated opposing duality of either promoting or retarding tumour growth. Manipulating this seemingly dynamic interaction for therapeutic benefit is a hotbed of much research. Recent findings in tumour immunology (both preclinical and clinical) build on more than a century of insights and provide a way forward to improving patient outcomes, long term survival and the predictability of "cures". This opinion piece attempts to summarise some of these historical and contemporary insights with a view to describing eminently testable therapeutic solutions.
\end{abstract}

Keywords: Tumour microenvironments, immune suppression, regulatory T cells, immune modulation, reversal, plasticity, spatio-temporal

\section{INTRODUCTION}

In 1926, Morris Fishbein, MD, the editor of the Journal of The American Medical Association (JAMA), wrote, "Cancers must be treated early, once a cancer has spread to surrounding tissue all attempts to halt the disease are usually hopeless" ${ }^{\prime[1]}$. 
In 2008, the United States Presidential Report on Cancer stated, "The toll of cancer has become simply an awful part of life; incidence is rising for several cancers; the most intransigent of malignancies remain impervious to treatment; (and) an absolute cure remains elusive". The report further added, "Despite declaring a national war on cancer in 1971 and investing many billions of dollars since then to understand and defeat cancer, our success against the disease in its many forms has been uneven and unacceptably slow" ${ }^{\text {"[2]. }}$. Previously in 1996, Director of the Pittsburgh Cancer Centre Michael Lotze said, "Mono and multi-agent chemotherapy just do not work in many settings, we should have dispensed with these notions years ago" ${ }^{[3]}$. This echoed the sentiment in Scientific American in a previous 1994 article entitled A War Not Won "Despite dramatic scientific gains, cancer remains an undaunted killer"[4].

Clearly, these sorts of concerns led Laurence Baker, MD, senior US oncologist and Chairman of the Southwest Oncology Group (SWOG) to state in 2010 in the Journal of the National Cancer Institute (JNCI) “ A cure is the expectation of society, we are not taking that seriously enough; We have a system that doesn't even really try to meet that expectation; I am trying to get people to stop saying how successful the cancer research enterprise is. It is not true. It is just not true" ${ }^{\text {[5] }}$.

Even today, in the midst of a "transformative" immunotherapy era of checkpoint inhibitor therapy, only a minority of patients derive benefits and at the cost of substantial biological and financial toxicity. Despite these negative and troubling historic comments, there is room for optimism.

\section{"Nature often gives us hints to her profoundest secrets" - William B Coley ${ }^{[6]}$}

For more than a century and since William Coley and his bacterial toxins, the notion that the immune system can be harnessed to treat cancer has met with limited and sporadic clinical success and much controversy ${ }^{[7]}$. And like cancer therapy today, it has been hit and miss. Those occasional spectacular "miracles" under various "immune tweaking" treatment modalities has intrigued clinicians and scientists alike and fuelled the relentless enthusiastic pursuit to make these random minority of successes and long term survival a reality in most patients. How can we translate/duplicate/amplify the efficacy at least seen in the mouse experiments? A broad explanation of why these sporadic successes occur infrequently was articulated by Prof Lloyd Old in 1993.

“

Why hasn't Coley's approach been forged into a widely available therapy with a predictable benefit for cancer patients? The best reason is, - the cellular and molecular language of inflammation and immunity had to be understood before the forces that Coley unleashed could be predictably translated into tumor cell destruction". - Lloyd Old $1993^{[7]}$.

His words back then actually described the way forward, detailed aspects of which are in the process of being elucidated today for clinical utility. Clearly, the correct sequence of therapeutic events already happens, at least randomly in some patients, even with the crudest approaches such as those used by Coley. The fundamental understanding (language) of the intimate interactions of the tumour with the immune system, particularly in the tumour microenvironment (TME), is providing answers to why some very different modalities can work effectively but only occasionally.

\section{The cellular and molecular language of inflammation and immunity}

A major advance in recent years has been the realisation that the immune system is not ignorant to the presence of cancer, and in particular, the cellular interactions of the TME collectively are "shielding" the cancer from immune destruction ${ }^{[8]}$. Specifically, the immune system is suppressing itself, tolerant to the 
growing tumour burden and creating substantial multilayered barriers to therapeutic success ${ }^{[9]}$. Remove or interfere with the mechanistic components of these immune circuits; these barriers can be broken down and can lead to tumour destruction, complete responses and improved survival ${ }^{[10]}$. Arguably, the most important critical lesson learnt with respect to the TME induced immune suppression is the realisation that this suppression has been caused by normal immune homeostasis. This homeostatic impasse is an intentional part of the way the immune system processes antigens and stops unwanted inflammation and damage to surrounding "normal" tissue. Importantly and recently, this impasse is being appreciated as being reversible ${ }^{[11]}$. Understanding a few simple rules of this homeostatic process has the potential to remove the "hit and miss" randomness that currently exists in cancer therapies.

\section{So who is the real enemy here, the cancer or the immune system?}

After decades of failure of tumour-centric approaches to cancer therapy, attention has gradually now turned to our immune system to do the "heavy lifting".

Irrespective of tumour morphology, patients with advanced cancer exhibit simultaneous immune activation and suppression ${ }^{[12]}$. While the TME consists of both cancerous and non-cancerous cells, most of these subpopulations cooperate synergistically to drive via positive feedback loops that are conducive to tumourigenesis. Further, the resultant local inflammatory environment appears to be a consistent component of malignant tumours and displays increasing concentrations of cytokines locally and systemically, particularly with rising disease burden ${ }^{[13]}$. Malignant tumours can significantly interfere with the patient's immune system, leading to fevers, paraneoplastic autoimmunity and sepsis ${ }^{[14,15]}$. Thus, despite a general environment of immune stimulation, evidence suggests that anti-tumour immune responses are being continuously attenuated, and this appears universal across the tumour types ${ }^{[16,17]}$.

The immune system has evolved the ability to recognise, destroy and remember foreign or corrupted peptides and antigens that are detrimental to our survival. Estimates suggest that the somatic hypermutation/recombination mechanisms to generate antigen-specific receptors in $\mathrm{T}$ cells can program for as many as $10^{15}$ possible unique receptors ${ }^{[18]}$. This diverse repertoire suggests that a functional immune system should be able to accommodate cancer adaptability and mutational drift. In support of the aforementioned, laboratory assays can detect $\mathrm{T}$ and $\mathrm{B}$ cell responses (autoantibodies etc.) to tumourassociated antigens and therapy-induced neoantigen ${ }^{[19-21]}$. Also, assays can detect attenuating antigenspecific regulatory $\mathrm{T}$ cell responses and indicative pro-inflammatory and immune-suppressive cytokines/inflammatory markers ${ }^{[22,23]}$.

\section{The immune cells of the TME and their function}

The TME can consist of a diverse immune cellular presence, including $\mathrm{T}$ and $\mathrm{B}$ lymphocytes, natural killer (NK), natural killer T (NKT) cells and regulatory T cells (Tregs). Tregs comprise diverse subsets of immunosuppressive cells that play critical roles in not only maintaining immune homeostasis and selftolerance, but also suppressing antitumour responses of cytotoxic lymphocytes. Other important cells include tumour-associated macrophages (TAMs) and myeloid-derived suppressor cells (MDSCS). All these cells can account for circa $10 \%$ of the total tumour cell population and can also be found in substantial concentrations within the tumour and in the tumour periphery. Collectively, they are major drivers of an immunosuppressive TME. TAMs exhibit both anti- and pro-tumoural effects. The high density of TAMs is a characteristic of most tumours and has been correlated to poor clinical outcomes ${ }^{[2-26]}$.

Within the TME, TAMs can polarise to M1-like pro-inflammatory interferon- $\gamma$ phenotype or the antiinflammatory/immunosuppressive M2-like phenotype, which induces the secretion of IL- 10 and TGF- $\beta$ to limit inflammation, enhances tissue repair, and promotes vascularisation. While these two TAMs are 
dominant, there is a high degree of plasticity and intermediaries. Macrophage M1/M2 polarisation appears to be transient, time and tissue-associated ${ }^{[27]}$. The M1/M2 phenotypes can shift in response to stimuli in the TME. As the "vanguard" antigen processor of the host immune response, TAMs offer the possibility to use their plasticity to modulate the underlying immune suppression, break tolerance and potentially improve clinical outcomes ${ }^{[28]}$. MDSCs can inhibit antitumour activities of T and NK cells and stimulate Treg, leading to tumour progression through the production of cytokines IL-10 and TGF- $\beta^{[29]}$.

In concert, all these aforementioned diverse immune cell types homeostatically maintain a "tumourfriendly" microenvironment both locally and systemically under a growing tumour burden. This all points to an underlying tightly controlled, dynamic "three-way conversation" between tumour cell populations, the pro-inflammatory and the immunosuppressive immune response circuits. Individually and collectively, these cells offer opportunities as therapeutic targets.

The positive and negative roles of cytokines within the immune response

Cytokines are produced by a plethora of immune other cells. Both APCs and T and B lymphocytes produce various cytokines cells in response to antigen processing and recognition. The amount of cytokine produced is dependent on the amount of antigen encountered and/or its antigenic potential. Interestingly, a number of specific cytokines play a major role paradoxically in both pro-inflammatory and immunosuppressive immune cellular pathways, either initiating or homeostatically terminating that immune response. In addition, another physiological/mechanistic insight is the transient actions of cytokine/receptor cellular interactions together with normal short half-life restrictions. This temporal aspect contributes to the ability of cytokines to have selective loco-regional/systemic effects ${ }^{[30-32]}$.

Three cytokines, in particular, are now appreciated to have opposing duality or bimodal attributes and have a long history in cancer immunotherapeutics. They are interleukin-2 (IL-2), interferon- $\gamma$ (IFN- $\gamma$ ) and tumour necrosis factor- $\alpha$ (TNF- $\alpha$ ). Normally, all have relatively short physiologic half-lives ${ }^{[33-35]}$.

This apparent temporal duality or paradox has confounded their clinical utility of knowing how to administer these cytokines (with respect to dose and duration) for the best clinical effect. In particular, IL-2 has an extensive 30-year history in treating advanced malignant melanoma (MM) and renal cell carcinoma RCC. In both incidences, on average, $\sim 7 \%$ of patients treated with IL-2 achieve complete responses and long-term survival after limited treatment ${ }^{[36]}$.

Known as the "master cytokine" IL-2, when this agent works successfully, it is clearly modulating an underlying/pre-existing tumour-specific immune response. The paradox arose in the mid-1990s when it was discovered that IL-2 also stimulates Tregs and can suppress an immune response $\mathrm{e}^{[37]}$. Thus, this provided an explanation for its limited and random efficacy. Similar duality opposing activity over Tregs was later elucidated for IFN- $\gamma$ and TNF- $\alpha^{[34,35]}$. More recently, similar attributes have been reported for PD-1 \& CTLA4 monoclonal antibodies (Mabs) as their targets are also expressed on Tregs, and blockade can cause tumour hyperprogression and self-limit efficacy ${ }^{[38,39]}$.

\section{Antigen load, recognition and tolerance induction - a critical insight}

Improved understanding of the immune system's role in cancer has reinvigorated research on the interplay between antigen load and immune tolerance induction. Indeed, elegant work by Gratz et al. ${ }^{[40]}$ and Pinheiro et al..$^{[41]}$ has shown that initial low levels of antigen promote Tregs and subsequently control the balance between T-effector lymphocytes \& Tregs and thus the balance between responsiveness and tolerance. This is a critically important insight into the fundamental nature of cancer immune suppression 
and antigen as a "prime mover" in the immune response. Moreover, this immune balance is mediated by an IL-2 centric feedback loop. Importantly, plasticity exists at the cytokine level between these two states ${ }^{[42,43]}$. A concept allergists are familiar with is the introduction of low dose antigens to promote tolerance to an otherwise vigorous response. There exist parallels with tumourigenesis, as presumably, cancer would start with a single cell with certain mutations and then proliferate, initiating a low tolerising dose of tumourassociated antigens (TAAs) ${ }^{[4]}$. The discontinuity theory of immunity, as articulated by Pradeu and Vivier ${ }^{[45]}$, Pradeu and Cooper ${ }^{[46]}$, and Matzinger's earlier danger signal hypothesis, provides a supporting theoretical framework to the experimental observations of Gratz et al. ${ }^{[40]}$ and Pinheiro et al. ${ }^{[41]}$. Together, this helps contextualise the influence of complex, slow, low-dose continuous TAA recognition and how sudden (perhaps therapy-induced) changes in antigen levels within the TME may subvert immune suppression and break tolerance in certain circumstances.

Further, interesting parallels in human pregnancy and cancer have also been drawn between the early immune response and subsequent induction of immune tolerance.

A number of studies have demonstrated that tumour and placenta tissue use the same mechanisms to suppress host immunity ${ }^{[4]}$. The immune privilege offered to develop neoplasms by Tregs mirrors that of a developing embryo, representing a highly effective and evolutionarily conserved immune tolerance mechanism that is co-opted by tumours. In midtrimester pregnancy and advanced cancer, systemic alterations in immunity are also detectable, particularly with respect to a helper T cell type 2 polarisation, $\mathrm{NK}$ induction of tolerogenic/angiogenic dendritic cells and NK permissiveness, despite low levels of major histocompatibility complex I expression ${ }^{[48]}$.

Another reproductive analogy in the context of cytokine cancer therapy is the introduction of the contraceptive pill. The "pill" came about as a result of detailed research and understanding of the menstrual cycle's orchestrated temporal dynamic interaction of reproductive hormones, their levels, cellular receptors and the various cell types in the female reproductive organ systems. Essentially, the effect of the pill (in part) was to "trick" the female physiology into thinking she was pregnant. This "trickery" was achieved by exogenously adding a little bit more of the same hormones (albeit now synthetic) into the system. This hormonal addition changed the "normal" sequence of events, modulated the reproductive homeostasis and allowed predictable and successful control of this dynamic system ${ }^{[4]}$.

\section{Tricking the system-disturbing immune homeostasis in the TME and breaking tolerance}

Numerous mouse models and some translational approaches have shown that tumour immune suppression following immune recognition is a significant obstacle to breaking immune tolerance ${ }^{[50]}$. Importantly and as argued above, this suppression/tolerance induction is an intentional antigen-specific process mediated by normal aspects of immune homeostasis and not that dissimilar to pregnancy? Consequently, taking advantage of our "new" understanding of immune homeostasis and its role in tumour establishment, maintenance, and progression, reveals promising therapeutic opportunities. Some of which are already available, and may require minor protocol modifications in order to substantially improve efficacy. From the immune modulation experience of the past 30 years, it has become apparent that there are several positions in the tumour immune circuitry that provide opportunities for selective therapeutic intervention points. These include: Treg ablation with chemo and radiotherapy, bolus cytokine therapy, checkpoint inhibitor monoclonal antibodies, intratumoural agents, or antigen load modification with various modalities ${ }^{[00-53]}$. The latter is discussed below. 


\section{Transmutability of antigen to cytokine conversion within and the adjacent TME}

Perhaps the simplest approach to immune modulation in order to break tumour tolerance appears to be using the TME's (and surrounding tissue's) own resident APCs to generate endogenous INF dominant cytokine production ${ }^{[54]}$. Causing sufficient tumour cell destruction and complex antigen production, at the same time preserving the nearby TME's APCs, has the potential via STING/ DAMP signalling pathways to break tumour tolerance locally and systemically ${ }^{[5]}$. A number of intratumoural strategies do the same thing; they supply a complex "soup" of antigen as a "burst" to the TME, which then is rapidly converted via APCs to INFs ${ }^{[5,57]}$. Modalities such as radiotherapy, high intensity focused ultrasound, radio wave ablation, cryotherapy, intratumoural food dye rose bengal, diterpene esters, all cause localised tumour cell destruction for immune processing and TME remodelling ${ }^{[58-61]}$. Similarly, it is now appreciated that the principal local and systemic mechanism of action of oncolytic viruses is caused by the lytic induced immune response against viral-infected cells ${ }^{[62]}$.

Ground-breaking clinical work by Tubin et al. ${ }^{[63]}$. and confirmed by Markovsky et al. ${ }^{[64]}$. in mouse experiments, demonstrated that systemic immune modulation and complete abscopal responses could be achieved by single/double dose partial tumour irradiation, particularly incorporating the hypoxic segment in the radiation field. Partial tumour hypofractionated (1-3 doses) irradiation preserves the TME's cellular immune signalling capabilities in the un-irradiated segment to do the "heavy lifting" locally and systemically via an ensuing antigen/cytokine-induced antitumour immune response $\mathrm{e}^{[63,64]}$.

\section{CONCLUSION}

Finally, our relatively recent understanding of the immune cellular components and interactions within the TME and their role in tumourigenesis and maintenance provides evidence on how to better use existing modalities to improve therapeutic outcomes. Further, these insights explain why earlier crude attempts at cancer immunotherapy worked occasionally/randomly and really not better than today's much more sophisticated varied attempts.

We now know the immune system is homeostatically suppressed, with the immune system tightly protecting the tumour burden from immune destruction. Normal inflammatory controls by loco-regional TAMs, Tregs, MDSCs, together with orchestrating cytokines such as IL2, INF- $\gamma$ and TNF- $\alpha$ involved in physiologic feedback loops, maintain the tumour friendly TME.

The prime mover in this suppression is the antigen load coming from the tumour. An apparent "normal" tissue in the eyes of the immune system, the tumour is not that dissimilar to the developing embryo.

We also know that introducing extra therapeutic cytokine into the intratumoural system can destabilise the status quo and thus interrupt "a natural course of events". Rather than attempting to immune modulate systemically and "deep" into the immune circuitry with toxic, expensive and inconsistent drugs, the simplest emerging and comprehensive solution may be to use therapeutic modalities that utilise the local TME APC machinery. Complex antigen loading via the STING/PAMPS pathways can induce endogenous cytokine production at sufficient levels to "throw a spanner in the works" of the underlying homeostatic suppression and break tolerance locally and systemically.

Further, evidence this can happen with various agents and modalities is appearing in the abscopal/bystander literature ${ }^{[6]}$. Clearly, the spatio-temporal influences of tumour-specific antigen load, the resultant cytokine production and the presence of opposing cell populations homeostatically balanced, are waiting to be used. In the face of a growing tumour burden, the system can be easily destabilised and thus break the 
homeostatic nature of tolerance. Put simply. It appears possible to use the TME's underlying immunology to be our best tumour-specific rational drug design factory.

\section{DECLARATIONS}

\section{Authors' contributions}

The author contributed solely to the article.

\section{Availability of data and materials}

Not applicable.

\section{Financial support and sponsorship}

None.

\section{Conflicts of interest}

The author declared that there are no conflicts of interest.

\section{Ethical approval and consent to participate}

Not applicable.

\section{Consent for publication}

Not applicable.

\section{Copyright}

(c) The Author(s) 2022.

\section{REFERENCES}

1. Fishbein M. Progress in medical science-IV. Scientific American 1926;134:26-7. DOI

2. The 2007-2008 President's Cancer Panel Report. Available from: https://deainfo.nci.nih.gov/Advisory/pcp/archive/pcp0708rpt/ExecSum.pdf [Last accessed on 25 Feb 2022].

3. Hall SS. Commotion in the blood. Henry Holt and Company. 1998. p. 249.

4. Beardsley T. A war not won. Scientific American 1994;270:130-8. DOI

5. Tuma RS. Large trials, small gains: is change on the way? J Natl Cancer Inst 2010;102:1216-17, 1223. DOI PubMed

6. Hall SS. Commotion in the blood. Henry Holt and Company. 1998 p. 30.

7. Hall SS. Commotion in the blood. Henry Holt and Company. 1998 p. 123.

8. Janssen LME, Ramsay EE, Logsdon CD, Overwijk WW. The immune system in cancer metastasis: friend or foe? J Immunother Cancer 2017;5:79. DOI PubMed PMC

9. Visser KE, Eichten A, Coussens LM. Paradoxical roles of the immune system during cancer development. Nat Rev Cancer 2006;6:2437. DOI PubMed

10. Burugu S, Dancsok AR, Nielsen TO. Emerging targets in cancer immunotherapy. Semin Cancer Biol 2018;52:39-52. DOI PubMed

11. Greten FR, Grivennikov SI. Inflammation and cancer: triggers, mechanisms, and consequences. Immunity 2019;51:27-41. DOI PubMed PMC

12. Lippitz BE. Cytokine patterns in patients with cancer: a systematic review. Lancet Oncol 2013;14:e218-28. DOI PubMed

13. Lippitz BE, Harris RA. Cytokine patterns in cancer patients: a review of the correlation between interleukin 6 and prognosis. Oncoimmunology 2016;5:e1093722. DOI PubMed PMC

14. Ihde DC. Paraneoplastic syndromes. Hosp Pract (Off Ed) 1987;22:105-12, 117. DOI PubMed

15. Maverakis E, Goodarzi H, Wehrli LN, Ono Y, Garcia MS. The etiology of paraneoplastic autoimmunity. Clin Rev Allergy Immunol 2012;42:135-44. DOI PubMed

16. Whiteside TL. Immune suppression in cancer: effects on immune cells, mechanisms and future therapeutic intervention. Semin Cancer Biol 2006;16:3-15. DOI PubMed

17. Stewart TJ, Smyth MJ. Improving cancer immunotherapy by targeting tumor-induced immune suppression. Cancer Metastasis Rev 2011;30:125-40. DOI PubMed

18. Davis MM, Bjorkman PJ. T-cell antigen receptor genes and T-cell recognition. Nature 1988;334:395-402. DOI PubMed

19. Macdonald IK, Parsy-Kowalska CB, Chapman CJ. Autoantibodies: opportunities for early cancer detection. Trends Cancer 2017;3:198-213. DOI PubMed

20. Wu J, Li X, Song W, et al. The roles and applications of autoantibodies in progression, diagnosis, treatment and prognosis of human 
malignant tumours. Autoimmun Rev 2017;16:1270-81. DOI PubMed

21. Schumacher TN, Schreiber RD. Neoantigens in cancer immunotherapy. Science 2015;348:69-74. DOI PubMed

22. Piersma SJ, Welters MJ, van der Burg SH. Tumor-specific regulatory T cells in cancer patients. Hum Immunol 2008;69:241-9. DOI PubMed

23. Chauhan R, Trivedi V. Inflammatory markers in cancer: potential resources. Front Biosci (Schol Ed) 2020;12:1-24. DOI PubMed

24. Hui L, Chen Y. Tumor microenvironment: sanctuary of the devil. Cancer Lett 2015;368:7-13. DOI PubMed

25. Anderson NM, Simon MC. The tumor microenvironment. Curr Biol 2020;30:R921-5. DOI PubMed PMC

26. Quail DF, Joyce JA. Microenvironmental regulation of tumor progression and metastasis. Nat Med 2013;19:1423-37. DOI PubMed PMC

27. Yuan R, Li S, Geng H, et al. Reversing the polarization of tumor-associated macrophages inhibits tumor metastasis. Int Immunopharmacol 2017;49:30-7. DOI PubMed

28. Poh AR, Ernst M. Targeting macrophages in cancer: from bench to bedside. Front Oncol 2018;8:49. DOI PubMed PMC

29. Kumar V, Patel S, Tcyganov E, Gabrilovich DI. The nature of myeloid-derived suppressor cells in the tumor microenvironment. Trends Immunol 2016;37:208-20. DOI PubMed PMC

30. Berraondo P, Sanmamed MF, Ochoa MC, et al. Cytokines in clinical cancer immunotherapy. Br J Cancer 2019;120:6-15. DOI PubMed PMC

31. Li L, Yu R, Cai T, et al. Effects of immune cells and cytokines on inflammation and immunosuppression in the tumor microenvironment. Int Immunopharmacol 2020;88:106939. DOI PubMed

32. Hoekstra ME, Vijver SV, Schumacher TN. Modulation of the tumor micro-environment by $\mathrm{CD} 8^{+} \mathrm{T}$ cell-derived cytokines. Curr Opin Immunol 2021;69:65-71. DOI PubMed PMC

33. Arenas-Ramirez N, Woytschak J, Boyman O. Interleukin-2: biology, design and application. Trends Immunol 2015;36:763-77. DOI PubMed

34. Zaidi MR. The interferon-gamma paradox in cancer. J Interferon Cytokine Res 2019;39:30-8. DOI PubMed PMC

35. Montfort A, Colacios C, Levade T, Andrieu-abadie N, Meyer N, Ségui B. The TNF paradox in cancer progression and immunotherapy. Front Immunol 2019;10:1818. DOI PubMed PMC

36. Coventry BJ, Ashdown ML. The 20th anniversary of interleukin-2 therapy: bimodal role explaining longstanding random induction of complete clinical responses. Cancer Manag Res 2012;4:215-21. DOI PubMed PMC

37. Sakaguchi S, Sakaguchi N, Asano M, Itoh M, Toda M. Immunologic self-tolerance maintained by activated T cells expressing IL-2 receptor alpha-chains (CD25). Breakdown of a single mechanism of self-tolerance causes various autoimmune diseases. J Immunol 1995; 155:1151-64. PubMed

38. Kamada T, Togashi Y, Tay C, et al. PD- $1^{+}$regulatory T cells amplified by PD-1 blockade promote hyperprogression of cancer. Proc Natl Acad Sci U S A 2019;116:9999-10008. DOI PubMed PMC

39. Marangoni F, Zhakyp A, Corsini M, et al. Expansion of tumor-associated Treg cells upon disruption of a CTLA-4-dependent feedback loop. Cell 2021;184:3998-4015.e19. DOI PubMed PMC

40. Gratz IK, Rosenblum MD, Maurano MM, et al. Cutting edge: self-antigen controls the balance between effector and regulatory T cells in peripheral tissues. J Immunol 2014;192:1351-5. DOI PubMed PMC

41. Pinheiro DF, Szenes-Nagy AB, Maurano MM, et al. Cutting edge: tissue antigen expression levels fine-tune $\mathrm{T}$ cell differentiation decisions in vivo. J Immunol 2020;205:2577-82. DOI PubMed PMC

42. Busse D, de la Rosa M, Hobiger K, et al. Competing feedback loops shape IL-2 signaling between helper and regulatory $\mathrm{T}$ lymphocytes in cellular microenvironments. Proc Natl Acad Sci U S A 2010;107:3058-63. DOI PubMed PMC

43. McNally A, Hill GR, Sparwasser T, Thomas R, Steptoe RJ. CD4+CD25+ regulatory T cells control CD8+ T-cell effector differentiation by modulating IL-2 homeostasis. Proc Natl Acad Sci U S A 2011;108:7529-34. DOI PubMed PMC

44. Challa DK, Mi W, Lo ST, Ober RJ, Ward ES. Antigen dynamics govern the induction of CD4(+) T cell tolerance during autoimmunity. J Autoimmun 2016;72:84-94. DOI PubMed

45. Pradeu T, Vivier E. The discontinuity theory of immunity. Sci Immunol 2016;1:AAG0479. DOI PubMed PMC

46. Pradeu T, Cooper EL. The danger theory: 20 years later. Front Immunol 2012;3:287. DOI PubMed PMC

47. Kareva I. Immune suppression in pregnancy and cancer: parallels and insights. Transl Oncol 2020;13:100759. DOI PubMed PMC

48. Bruno V, Corrado G, Baci D, et al. Endometrial cancer immune escape mechanisms: let us learn from the fetal-maternal interface. Front Oncol 2020;10:156. DOI PubMed PMC

49. Nottke A. Taming the cycle: how does the pill work? Available from: https://sitn.hms.harvard.edu/flash/2008/issue40/ [Last accessed on $25 \mathrm{Feb} 2022]$.

50. Flemming A. Cancer: tumour-specific ablation of Treg cells induces anticancer response. Nat Rev Drug Discov 2016;15:676-7. DOI PubMed

51. Ohue Y, Nishikawa H. Regulatory T (Treg) cells in cancer: can Treg cells be a new therapeutic target? Cancer Sci 2019;110:2080-9. DOI PubMed PMC

52. Boyman O, Kolios AG, Raeber ME. Modulation of T cell responses by IL-2 and IL-2 complexes. Clin Exp Rheumatol 2015;33:S54-7. PubMed

53. Tran E, Robbins PF, Rosenberg SA. 'Final common pathway' of human cancer immunotherapy: targeting random somatic mutations. Nat Immunol 2017;18:255-62. DOI PubMed PMC

54. Shimizu K, Iyoda T, Okada M, Yamasaki S, Fujii SI. Immune suppression and reversal of the suppressive tumor microenvironment. Int Immunol 2018;30:445-54. DOI PubMed 
55. Ahmed A, Tait SWG. Targeting immunogenic cell death in cancer. Mol Oncol 2020;14:2994-3006. DOI PubMed PMC

56. Vaes RDW, Hendriks LEL, Vooijs M, De Ruysscher D. Biomarkers of radiotherapy-induced immunogenic cell death. Cells 2021;10:930. DOI PubMed PMC

57. Storozynsky Q, Hitt MM. The impact of radiation-induced DNA damage on cGAS-STING-mediated immune responses to cancer. Int J Mol Sci 2020;21:8877. DOI PubMed PMC

58. McLaughlin M, Patin EC, Pedersen M, et al. Inflammatory microenvironment remodelling by tumour cells after radiotherapy. Nat Rev Cancer 2020;20:203-17. DOI PubMed

59. Xu W, Atkinson VG, Menzies AM. Intratumoural immunotherapies in oncology. Eur J Cancer 2020;127:1-11. DOI PubMed

60. Chavez M, Silvestrini MT, Ingham ES, et al. Distinct immune signatures in directly treated and distant tumors result from TLR adjuvants and focal ablation. Theranostics 2018;8:3611-28. DOI PubMed PMC

61. Yip YK, Pang RH, Oppenheim JD, et al. Stimulation of human gamma interferon production by diterpene esters. Infect Immun 1981;34:131-9. DOI PubMed PMC

62. Ribas A, Dummer R, Puzanov I, et al. Oncolytic virotherapy promotes intratumoral T cell infiltration and improves anti-PD-1 immunotherapy. Cell 2017;170:1109-19.e10. DOI PubMed PMC

63. Tubin S, Khan MK, Salerno G, Mourad WF, Yan W, Jeremic B. Mono-institutional phase 2 study of innovative Stereotactic Body RadioTherapy targeting PArtial Tumor HYpoxic (SBRT-PATHY) clonogenic cells in unresectable bulky non-small cell lung cancer: profound non-targeted effects by sparing peri-tumoral immune microenvironment. Radiat Oncol 2019;14:212. DOI PubMed PMC

64. Markovsky E, Budhu S, Samstein RM, et al. An antitumor immune response is evoked by partial-volume single-dose radiation in 2 murine models. Int J Radiat Oncol Biol Phys 2019;103:697-708. DOI PubMed PMC

65. Rodríguez-Ruiz ME, Vanpouille-Box C, Melero I, Formenti SC, Demaria S. Immunological mechanisms responsible for radiationinduced abscopal effect. Trends Immunol 2018;39:644-55. DOI PubMed PMC 